\title{
Optimization technics to identify the characteristics of a hydroformed structure
}

\author{
B. Radi ${ }^{1 a}$, A. Cherouat ${ }^{2}$, A. El Hami ${ }^{3}$, A. Mahfoudh ${ }^{4}$ \\ ${ }^{1}$ FST Settat, BP : 577, Route de Casablanca, Settat, Morocco \\ ${ }^{2}$ GAMMA3, UTT, 12 rue Marie-Curie, BP 2060, 10010 Troyes, France \\ ${ }^{3}$ LMR, INSA de Rouen, BP: 8, Avenue de l'Université, 76801 St Etienne de Rouvray, France \\ ${ }^{4}$ LMSSDT, ESSTT 5 Av Taha Hussein, Montfleury 1008, Tunisia
}

Received 10 December 2009, Accepted 15 February 2010

\begin{abstract}
This paper presents an experimental/numerical methodology which aims to improve 3D welded tube considering their anisotropic effect, geometrical singularities found in the welded joint, and heat affected zone behaviour by hydroforming process. This process contributes to reduce the number of welding and assembly operations needed to generate complex structures, while improving the weight saving and quality of finished parts. In spite of the advances in the performance of this manufacturing technology, some problems are linked to particularities of certain raw materials and additional studies must be developed, like in the case of welded tubes. The experimental study is dedicated to the identification of stress-strain flow of the base metal and the heat-affected zone from the global measure of tube displacement, thickness evolution and internal pressure expansion. Nanoindentation test is adapted to investigate the heat affected zone mechanical behaviour. Workpiece behaviour's models used to simulate the expansion tests, made it possible to highlight the combined effects of the inhomogeneous behaviour of basic material and heat affected zone, as well as the geometrical singularities found in the welded tubes. From the simulations carried out, it is clear the influence of the plastic flow behaviour of the welded tube in the final results (thickness distribution, stress instability, tube circularity, critical thinning and rupture).
\end{abstract}

Keywords: hydroforming, welded tube, anisotropic plastic flow, nanoindentation, numerical simulation

\section{Introduction}

Due to the increasing demands for the manufacturing of complex and lightweight parts in various fields, the hydroforming process of welded tubes (WT), considered as an alternative to stamping for obtaining the hollow tubular structures, occupies an increasingly significant place in automotive aircraft and aerospace industries [1-3].

However, the effective use of the hydroforming technology in the manufacturing industry has triggered new challenges in terms of the technological parameters specified for the forming process and particularly the material properties of the WT [4-5].

The latest studies are devoted to the mechanical and numerical modelling of the hydroforming processes using the finite element analysis [6-10], allowing the prediction of the material flow and optimisation of hydroforming parameters [11-19]. In addition, the accuracy of the numerical

\footnotetext{
${ }^{\text {a }}$ Corresponding author: bouchaib.radi@yahoo.fr
}

prediction remains linked to the fidelity models representing the behaviour of the WT and particularly the mechanical properties (plasticity metal flow, anisotropy effect and damage initiation and growth) of the base metal (BM) and the welded joint (WJ) zone [20-21]. The characteristic of the welded structures comes from the significant transformations (shaping, calibration, bending, rolling, welding...) undergone by the tube throughout its production cycle. All these operations induce complex evolutions of hardening flow and material anisotropy; those are amplified by the presence of the WJ along the tube. This material imperfection is characterized by a heat affected zone (HAZ) singular by its geometry and properties. This fact shows that oriented tensile tests are not adapted to identify the total behaviour of the tubes with regard to the loading paths generated by the hydroforming process [22-23].

This work presents a computational based numerical and experimental methodology devoted to the numerical simulation of WT formability. The experimental study is dedicated to the identification of material properties (in BM and HAZ) from the global measure of thickness 
evolution, radial displacement and tube expansion. In the numerical simulation, two types of behaviour (homogenous and inhomogeneous) are proposed to model the WT behaviour.

In the homogenous case, free expansion tests are carried out on fixed ends tubular test-tubes subjected to an internal pressure, are exploited to determine the hardening model of BM and HAZ as well as the geometrical singularities found in the WT. In the inhomogeneous case, the BM part and the HAZ are characterized by different plastic strain flow. The base metal zone is modelled using isotropic elastoplastic behaviour (Swift model) while the HAZ is modelled by analyzing indentation load-depth curves to evaluate stressstrain flow. Applications are made to the simulation of the WT hydroforming to show the efficiency of the proposed methodology.

The confrontation between the numerical (estimates for the two approaches) and experimental results has proved to be of great tool to the improvement and optimization of WT formability.

\section{Experimental procedure}

The swelling tests carried out until bursting showed that all the fissures are initiated on the central area of the expanding zone not far from the weld zone (see Fig. 3). The defect pressure recorded is ranged between 30.5 and $32 \mathrm{MPa}$. This failure of WT can be attributed to diverse causes. The present work is specially focused on the study of (i) geometrical singularities found in the WJ and (ii) the inhomogeneous behaviour of the base metal and the HAZ of the tube formability.

The weld tubes made of stainless steel (AISI $304)$ has $250 \mathrm{~mm}$ initial length, $48 \mathrm{~mm}$ inner diameter and $49.2 \mathrm{~mm}$ outer diameter $(1.2 \mathrm{~mm}$ initial thickness). The controlled process parameters are the axial load of the two ends of the tube fixed and the internal fluid pressure is applied as a uniformly distributed load to the tube inner surface and is introduced as a linearly increasing function of time. Comparator is used to measure the external diameter of tubular parts in the middle of their length. Seventeen samples have been tested until bursting step. For each of the tested tubes, the bulge height, the radius of curvature along the longitudinal direction and the wall thickness were measured. The schematic configuration of the free expansion test is shown in figure 1. Five representative experiments were made during hydroforming tests. The bursting pressures recorded, are ranged between 30.5 and
$32 \mathrm{MPa}$ so that the weld properties may have a significant effect on the deformation and the formability of the tube blank.

\section{Welded zone}
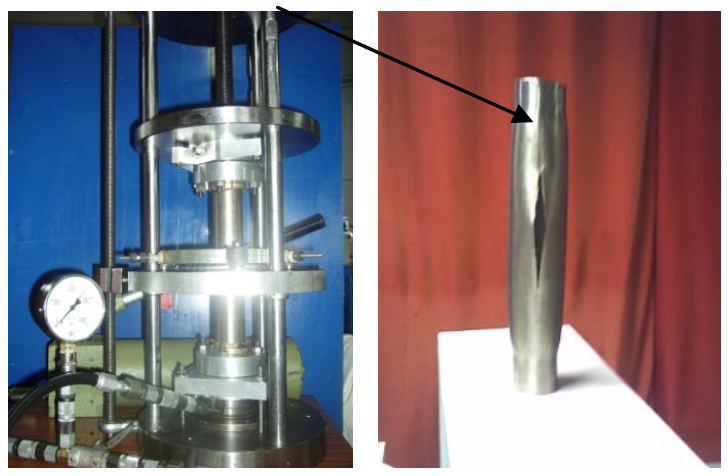

Fig. 1. Experimental welded tube hydroforming

The ultrasonic technique is very useful for nondestructive measurement of the mechanical properties of materials. It based an ultrasonic source to measure the wall thickness variation by transmission of wave mechanics in the material through a piezoelectric transducer. The transducer is placed on each point of the grid tube and the values are directly displayed on the screen of the device and stored with an accuracy of a thousandth $(0.001 \mathrm{~mm})$. The measurements obtained to estimate the tube thinning along the middle contour and the axis line. On the tube contour, nine measures of the thinning are made using ultrasound, and fluctuation around an average value of about 0.855 is deduced.

In recent years, indentation techniques have been used extensively to determine directly the material hardness or, indirectly, the bulk mechanical properties when other methods have proven to be unsuitable. In particular, as indentation techniques have improved, nanoindentation has become the most popular method for studying the mechanical properties of thin films, joints and coatings.

Compared to the base metal, the volumic fraction of the HAZ or the welded joint is relatively weak; consequently, the contribution of the WJ would be unperceivable with radial displacement measurements on test-tubes (global approach). Thus it is essential to use not classic techniques to characterize the local material properties. That justifies nanoindentation tests exploitation in order to determine the local behaviour of the weld tube. In this study, the 
nanoindentation with a pyramidal BERKOVICH indenter has been used as an effective tool to characterize the mechanical properties of welded joint and HAZ.

The geometrical singularities introduced by the WJ are revealed by scanning optical electronic microscope on the cross section of the wall. This geometrical defect modifies the wall thickness as observed on the notch profile on the tube internal wall as shown on Fig. 2. The measured dimensions of this notch indicate an average depth of about $0.115 \mathrm{~mm}$ and a width varying between 3.0 and $4.2 \mathrm{~mm}$.

Results obtained from twenty indents on the HAZ (Fig. 3) show that the relative variations recorded between the representative points $\mathrm{A}, \mathrm{B}$ and $\mathrm{C}$ do not exceed $15 \%$. It allows admit that mechanical properties of the HAZ are homogeneous on few microns depths.

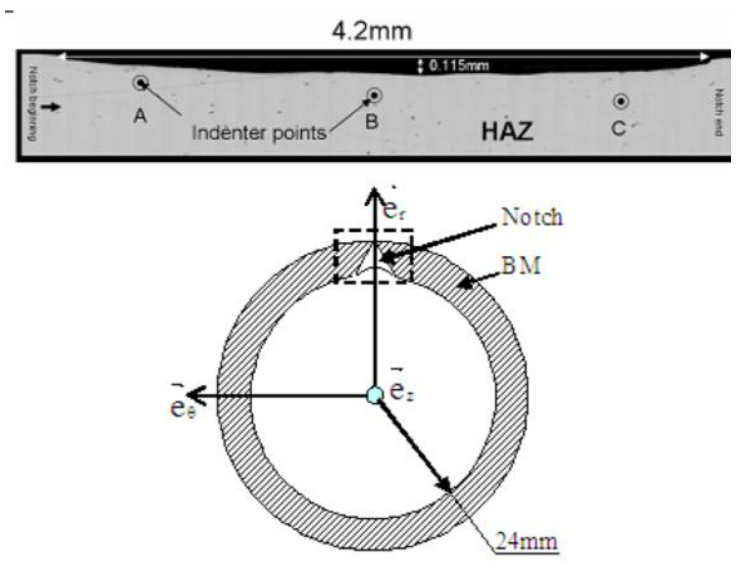

Fig. 2. Optical image of the cross-section and indents location points in HAZ

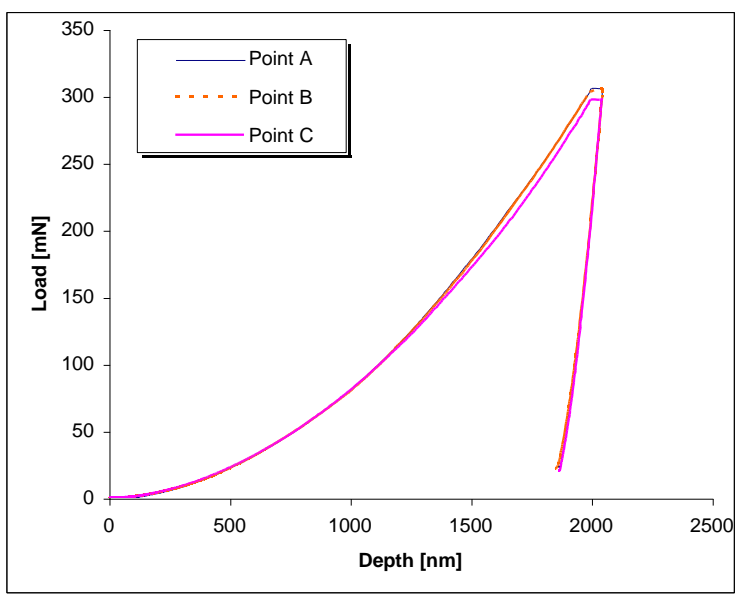

Fig. 3. Experimental depth-indentation force graphs of representative indents A, B and C.

\section{Numerical modelling}

\subsection{Mechanical characteristic of welded tube behaviour}

Taking into account the ratio thickness/diameter of tube, the radial stress is considerably small compared to the circumferential $\sigma_{\theta}$ and longitudinal stresses $\sigma_{\mathrm{z}}$. In addition, the principal axes of the stress tensor and the orthotropic axes are considered coaxial. The transverse anisotropy assumption represented through the yield criterion proposed by Hill 1950 can be written as:

$$
\bar{\sigma}^{2}=F\left(\sigma_{z}-\sigma_{\theta}\right)^{2}+G \sigma_{z}{ }^{2}+H \sigma_{\theta}{ }^{2}(1)
$$

with $(\mathrm{F}, \mathrm{G}, \mathrm{H})$ are the parameters characterizing the current state of anisotropy

If the circumferential direction is taken as a material reference, the anisotropy effect can be characterized by a single coefficient $R$ [17] and the equation (1) becomes:

$$
\bar{\sigma}^{2}=\frac{1}{1+R}\left[R\left(\sigma_{z}-\sigma_{\theta}\right)^{2}+\sigma_{z}{ }^{2}+\sigma_{\theta}{ }^{2}\right](2)
$$

The assumptions of normality and consistency lead to the following equations:

$$
\left\{\begin{array}{l}
\mathrm{d} \varepsilon_{\theta}=\frac{\mathrm{d} \bar{\varepsilon}}{\bar{\sigma}}\left(\sigma_{\theta}-\frac{R}{1+R} \sigma_{z}\right) \\
\mathrm{d} \varepsilon_{z}=\frac{\mathrm{d} \bar{\varepsilon}}{\bar{\sigma}}\left(\sigma_{z}-\frac{R}{1+R} \sigma_{\theta}\right)
\end{array}\right.
$$

where $\bar{\varepsilon}$ is the effective plastic strain and $\left(\varepsilon_{\theta}, \varepsilon_{\mathrm{z}}\right)$ are the strains in the circumferential and the axial directions.

The effective strain for anisotropic material can be derived from equivalent plastic work definition, incompressibility condition, and the normality condition:

$$
\begin{aligned}
& \mathrm{d} \bar{\varepsilon}=\frac{\sqrt{1+R}}{\sqrt{1+2 R}} \sqrt{\mathrm{d} \varepsilon_{\mathrm{z}}^{2}+\mathrm{d} \varepsilon_{\theta}^{2}+R\left(\mathrm{~d} \varepsilon_{z}-\mathrm{d} \varepsilon_{\theta}\right)^{2}} \\
& =\left(\sqrt{\gamma^{2}+\frac{2 R}{1+R} \gamma+1}\right) \frac{1+R}{\sqrt{1+2 R}} \mathrm{~d} \varepsilon_{\theta}
\end{aligned}
$$

with $\gamma=\frac{\mathrm{d} \varepsilon_{z}}{\mathrm{~d} \varepsilon_{\theta}}$

Taking into account the relations expressing strain tensor increments, the equivalent stress (eq. 2) becomes: 


$$
\bar{\sigma}=\left(\sqrt{1+\gamma^{2}+\frac{2 R}{1+R} \gamma}\right) \frac{\sqrt{1+2 R}}{1+R+R \gamma} \sigma_{\theta}(5)
$$

In the studied case, the tube ends are fixed. As a consequence, the longitudinal increment strain $d \varepsilon_{\mathrm{z}}=0$, and then relations (4) and (5) become:

$$
\bar{\sigma}=\left(\sqrt{\frac{2 R^{2}+3 R+1}{(1+R)^{3}}}\right) \sigma_{\theta} ; \mathrm{d} \bar{\varepsilon}=\left(\frac{1+R}{\sqrt{1+2 R}}\right) \mathrm{d} \varepsilon_{\theta}(6)
$$

The knowledge of the two unknown strain $\varepsilon_{\theta}$ and stress $\sigma_{\theta}$ needs the establishment of the final geometrical data linked to the tube (diameter and wall thickness):

$$
\varepsilon_{\theta}=\ln \left(\frac{d}{d_{0}}\right) \text { and } \sigma_{\theta}=\frac{P d}{2 t}
$$

where $\mathrm{P}$ is the internal pressure, $\left(\mathrm{d}, \mathrm{d}_{0}\right)$ are the respective average values of the current and initial diameter of the sample and ( $\mathrm{t}$ ) is the current wall thickness obtained according to the following relation:

$$
t=t_{0} \mathrm{e}^{-(1+\gamma) \varepsilon_{\theta}}
$$

Finally, the material characteristics of the tube (base metal) are expressed by the effective stress and effective strain according to the following equation (Swift model):

$$
\bar{\sigma}=K\left(\varepsilon_{0}+\bar{\varepsilon}\right)^{n}
$$

The values of the strength coefficient $\mathrm{K}$; the strain hardening exponent $\mathrm{n}$; the initial strain $\varepsilon_{0}$ and the anisotropic coefficient $\mathrm{R}$ are identified numerically using simplex optimization algorithm (Matlab). For the determination of the stress-strain relationship using bulge test, the radial displacement, the internal pressure and the thickness at the center of the tube are required.

A three dimensional finite element analysis (FEA) has been performed using the finite element code Abaqus to investigate the effect of the geometrical singularities of the WJ and the inhomogeneous behavior of the BM and the HAZ on the tube formability. The model uses tri-linear hexahedral eight-node elements in modeling the initial tube. Special fine mesh is used in the weld zone area. For validation, comparisons between numerical and experimental results are done. They concern the hardening laws (homogenous and inhomogeneous), the anisotropy effect, the wall thickness and the thickness variation along length and middle circumference of bulged part.

\subsubsection{Homogenous behaviour with geometrical singularities}

In this case, the BM with geometrical singularities found in the WT is supposed orthotropic transverse, whereas its behaviour is represented by Swift model. The optical microscope observation on the cross section of the wall is used to build the geometrical profile of the notch generated by the welded junction (see Fig.4).

By considering the assumptions relating to an isotropic thin shell $(\mathrm{R}=1)$ with an uniform thickness, the previously established relations (6), (7) and (9) allow to build the first experimental hardening model using measurements of internal pressure/radial displacements. This model is then proposed, as initial solution, to solve the inverse problem of required hardening law that minimizes the following objective function:

(7)

$$
\xi_{\mathrm{F}}=\frac{1}{m_{p}} \sqrt{\sum_{i=1}^{m_{p}}\left(\frac{F_{\mathrm{exp}}^{i}-F_{\mathrm{num}}^{i}}{F_{\mathrm{exp}}^{i}}\right)}
$$

where $F_{\exp }^{i}$ is the experimental value of the thrust force corresponding to $i^{\text {th }}$ nanoindentation depth $\mathrm{H}_{\mathrm{i}}, \quad F_{\text {num }}^{i}$ is the corresponding simulated thrust force and $\mathrm{m}_{\mathrm{p}}$ is the total number of experimental points.

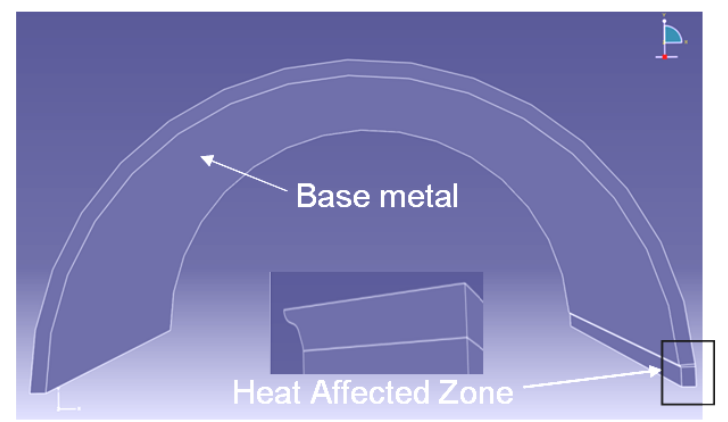

Fig. 4. Profile of the geometrical singularities of the heat affected zone

Different flow stress evolutions of isotropic hardening (initial, intermediate and optimal) are proposed in order to estimate the best behaviour of the BM with geometrical singularities found in the WT. Figs. 5 and 6 show the effective stress versus plastic strain curves and the associate pressure/radial displacement for these three cases. As it can be seen, there is a good correlation between the optimal evolution of Swift hardening and the experimental results. Table 1 summarizes the parameters of these models. 
Table 1: Swift parameters of different hardening evolution

\begin{tabular}{|l|c|c|c|}
\hline \multicolumn{1}{|c|}{$\begin{array}{c}\text { Hardening } \\
\text { model }\end{array}$} & $\varepsilon_{0}$ & $\begin{array}{c}\mathrm{K} \\
(\mathrm{MPa})\end{array}$ & $\mathrm{n}$ \\
\hline Initial & 0.025 & 1124.6 & 0.2941 \\
\hline Intermediate & 0.055 & 692.30 & 0.2101 \\
\hline Optimal & 0.080 & 742.50 & 0.2359 \\
\hline
\end{tabular}

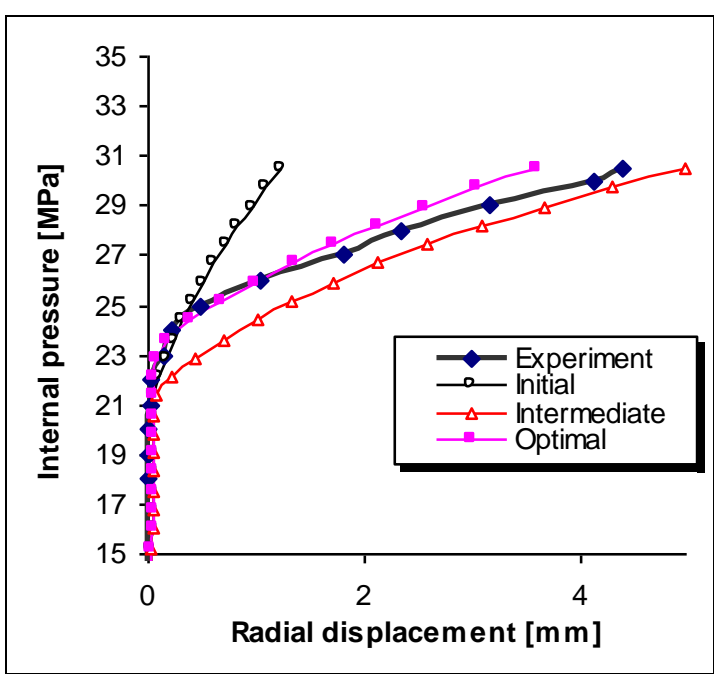

Fig. 5. Internal pressure versus radial displacement

The anisotropy factor $\mathrm{R}$ is determined only for the optimal hardening evolution. In the problem to be solved there is only one parameter which initial solution exists, that it corresponds to the case of isotropic material $(\mathrm{R}=1)$.

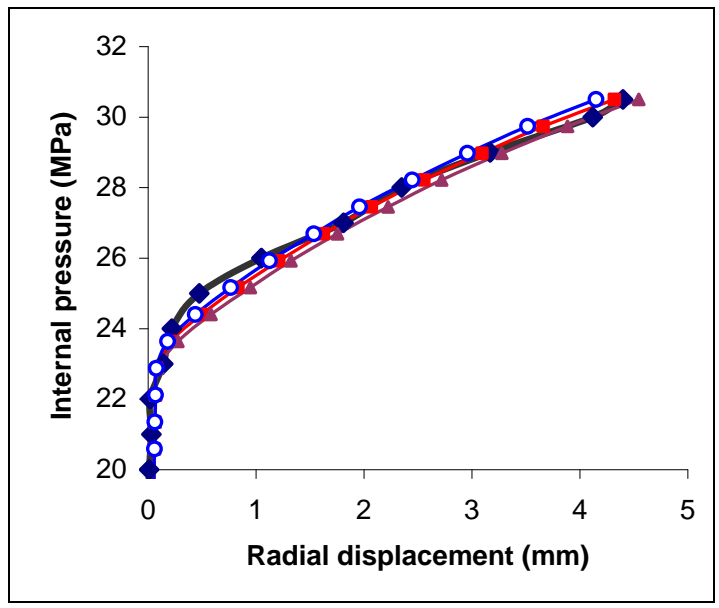

Fig. 6. Radial displacement for different values of anisotropy coefficient $\mathrm{R}$

The numerical iterations were performed on the WT with non-uniformity of the thickness and the obtained results are shown in figure 6. A good improvement in the quality of predicted results is noted if $\mathrm{R}$ corresponding the value of 0.976 .

\subsection{Hydroforming with anisotropic behaviour}

\subsubsection{Localization of the bursting zones}

The comparison between the experimental results and the numerical prediction, using the identified model (Table 1), emphasized the effects of the geometrical singularities of $\mathrm{WJ}$ as well as the mechanical characteristics of the HAZ on the WT formability. Indeed, it seems that the geometrical singularities of the $\mathrm{WJ}$ are not the single indicator which locates the bursting zones. Fig. 7 shows, for the same profile of geometrical singularities, the effects of the inhomogeneous mechanical properties of BM and HAZ, on the initiation and growth of ductile rupture. Initially, the sample inflated so that the test section remained uniform. However, eventually it developed an axisymmetric bulge followed by localized thinning along a generator of tube in the $\mathrm{WJ}$ that led to ductile rupture.
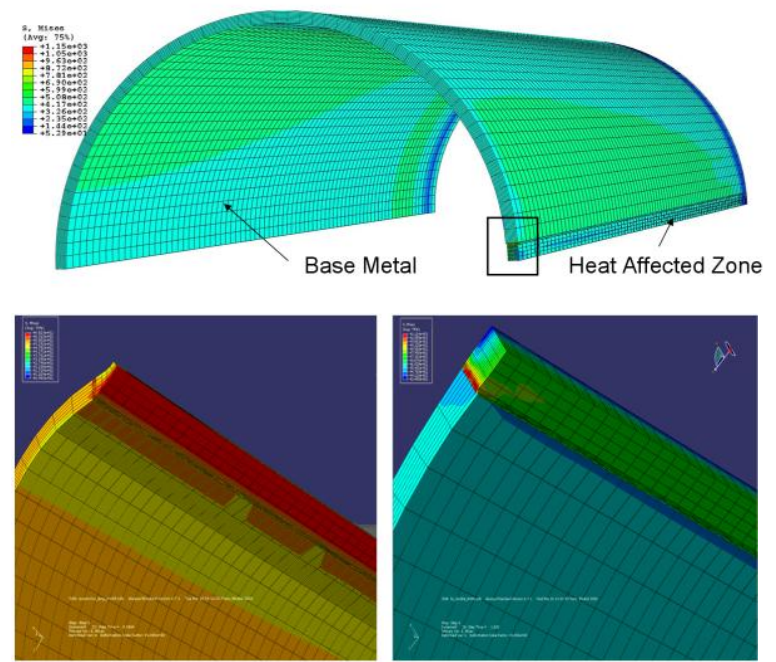

Fig. 7. Effective stress in BM and HAZ

(a) homogeneous, (b) inhomogeneous

Fig. 8 illustrates the profile of the effective stress (for $\mathrm{R}=0.965$ and $\mathrm{R}=1.035$ ) on the internal half contour in the symmetry plane $\mathrm{z}=0$ of homogenous and inhomogeneous case. For this result the local maximum effective stress values is $700 \mathrm{MPa}<\bar{\sigma}_{\max }<820 \mathrm{MPa}$. These values are significantly larger than the average stress at failure illustrating the local nature of the failure. If 
the characteristics of the HAZ and the BM are the same (homogeneous), the instability, as indicated by attainment of a stress maximum (of about $700 \mathrm{MPa})$ is located at the medium of the tube (angular position $0^{\circ}$ ) and concentred along the $\mathrm{WJ}$ thickness (approximately $3^{\circ}$ ). However, the inhomogeneous behaviour of the tube and the geometrical singularities found in the $\mathrm{WJ}$, produce an irregular stress profile on $9 \mathrm{~mm}$ width, the maximum stress (of about $800 \mathrm{MPa}$ ) corresponds to $3^{\circ}$ angular position on $\mathrm{BM} / \mathrm{HAZ}$ interface. The effect of the material anisotropy is very negligible.

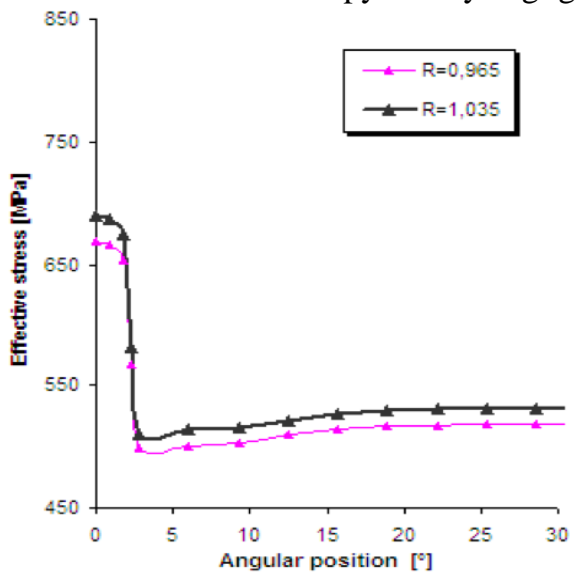

a): homogeneous

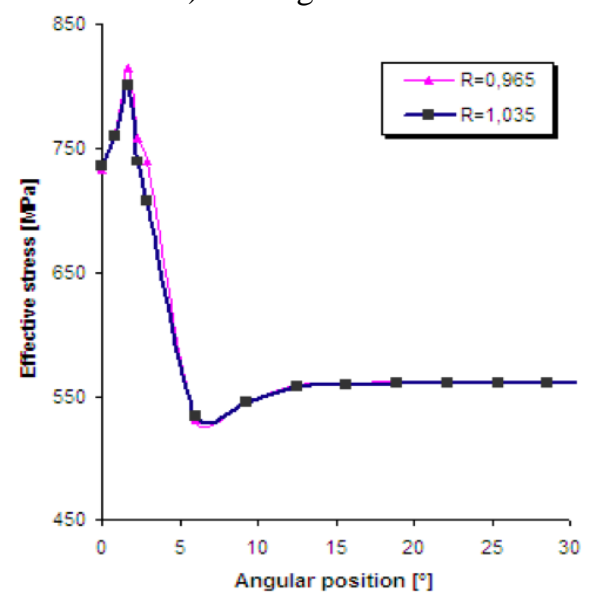

b): inhomogeneous

Fig. 8. Equivalent stress profile in interior central contour (a): homogeneous (b): inhomogeneous

\subsubsection{Tube circularity after expansion}

Among the hydroforming process advantages it's necessary to enumerate the high aesthetic quality and dimensional tolerances of the finished parts. Hydraulic free expansion similar to the HP is likely to provide indicators on the problems incurred by the WT during a shaping cycle. For this reason, the combined effects of the geometrical singularities and the inhomogeneous of the imposed behaviour on the circularity of the expanded WT are examined.

Fig. 9 shows, in both cases homogenous and inhomogeneous WT properties $(\mathrm{R}=0.965$ and $1.035)$, the profile of radial displacement at the central contour relating to $30 \mathrm{MPa}$ internal pressure. It is noted that, the weakest variations of radius are located in the WJ. The relative circularity defect defined by the ratio circularity/initial radius is more significant for homogenous case (HAZ and BM have the same mechanical properties), ranging between 3.2 and $7.7 \%$. This defect varies from 2.1 to $2.5 \%$ for inhomogeneous case. In addition, whether BM and HAZ are homogeneous or not, the maximum variations correspond to the 44.7 and $134.9^{\circ}$ angular positions; the minimal ones are located at 0,93 and $180^{\circ}$ angular positions. Table 2 recapitulates the numerical radial displacements.

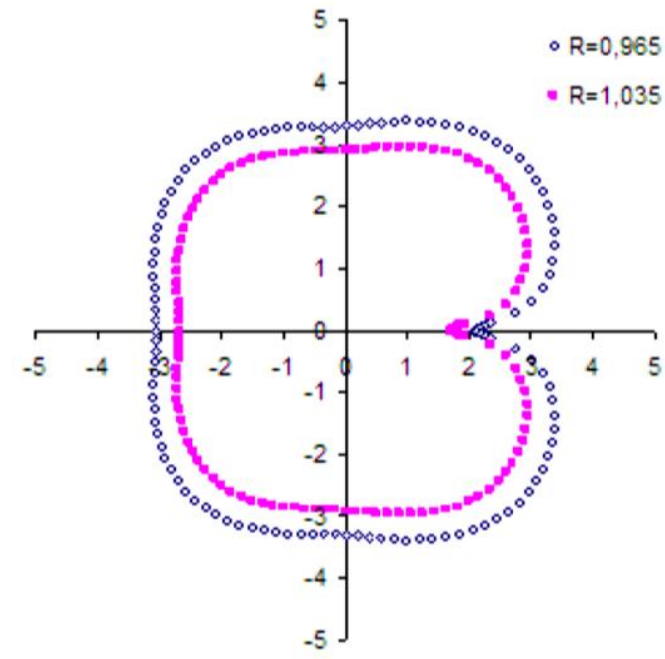

a) homogeneous case

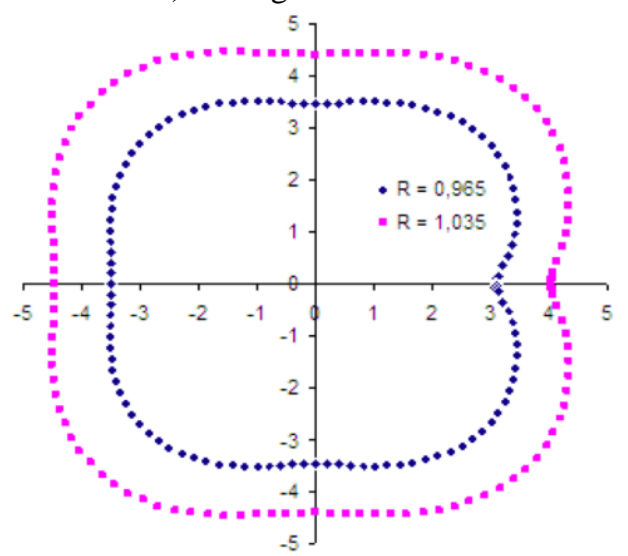

b) inhomogeneous case

Fig. 9. Numerical cross section shape at the centre of the tube (a) homogeneous (b) inhomogeneous 
Table 2: Numerical data characteristics of the radial displacements profile

\begin{tabular}{|c|c|c|c|c|c|c|}
\hline & \multicolumn{4}{|c|}{ Radial displacement (mm) } & \multirow[b]{2}{*}{$\begin{array}{c}\text { Circularit } \\
\text { y }(\mathrm{mm})\end{array}$} \\
\hline $\begin{array}{l}\text { MB/ } \\
\text { HAZ }\end{array}$ & $R$ & average & Max & $0^{\circ}$ & $180^{\circ}$ & \\
\hline \multirow{2}{*}{$\begin{array}{c}\text { homoge } \\
\text { neous }\end{array}$} & 0.965 & 3.42 & 3.92 & 2.02 & 3.06 & 1.90 \\
\hline & 1.035 & 3.05 & 3.45 & 1.68 & 2.65 & 0.80 \\
\hline \multirow{2}{*}{$\begin{array}{l}\text { inhomo } \\
\text { geneous }\end{array}$} & 0.965 & 3.70 & 4.01 & 3.10 & 3.49 & 0.52 \\
\hline & 1.035 & 4.71 & 5.08 & 4.04 & 4.45 & 0.63 \\
\hline
\end{tabular}

\subsubsection{Tube thinning}

The hypothesis relating to the plastic incompressibility condition and the homogenous thickness strains allow thanks to local measurement of longitudinal and circumferential strains to evaluate the wall thinning. For a constant ratio of strain $\gamma$ (Eq.4), relations (7) and (9) provide an estimate of thickness evolution in the transverse central zone ( $\gamma$ near to zero).

Consequently, from the measured values of radial displacement, it is possible to deduce directly the current thinning. To generalize this method to other test-tube zones, the measurement of longitudinal strain becomes necessary. In addition, a direct measurement thickness is possible using an ultrasonic source. This method is applied by its good performance for checking the coating thicknesses.

Fig. 10 compares the experimental results with the numerical prediction (with homogenous and inhomogeneous behaviours) of thinning along the middle circumference. The thickness ratio $t / t_{0}$ measured by ultrasound method, compared to the initial thickness is characterized by fluctuations around an average value of 0.855 . It can be noted that, the thinning is uniform with varying averages from 0.81 to $0.88 \mathrm{~mm}$.

The irregularities are limited to the singularities imposed by the WJ. The fluctuations of ultrasound measurements could be attributed to the positioning defects of the ultrasonic transmitter and/or to the equipment resolution degree. However, the variations observed between prediction and measurements let admit that results of identified models are coherent with experimental results.

Figure 11 compares the experimental results with the numerical prediction (inhomogeneous behaviour) of thickness along length of bulged part of the tube. The maximum thinning is located at the central part of the WT (around an average value of 0.855 ).

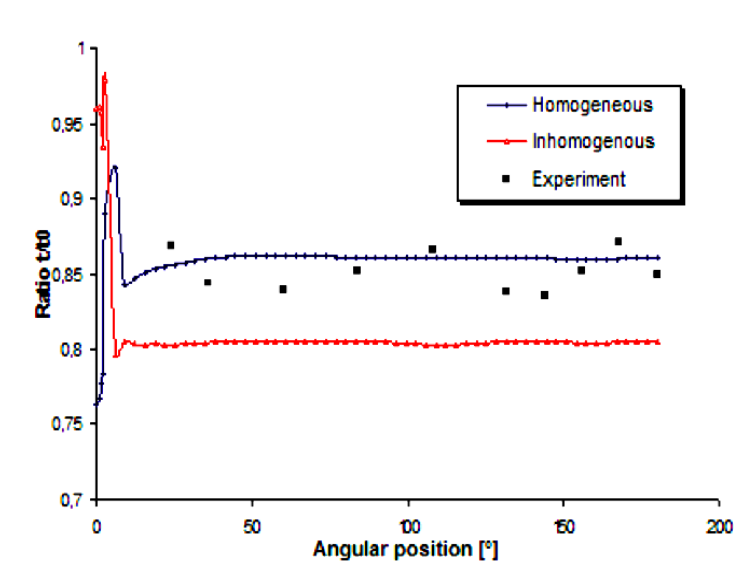

Fig. 10. Thinning along middle cirumference

Between ultrasonic measurements and numerical prediction, the differences related to the thinning expressed by the ratio of thickness, are below $6 \%$.

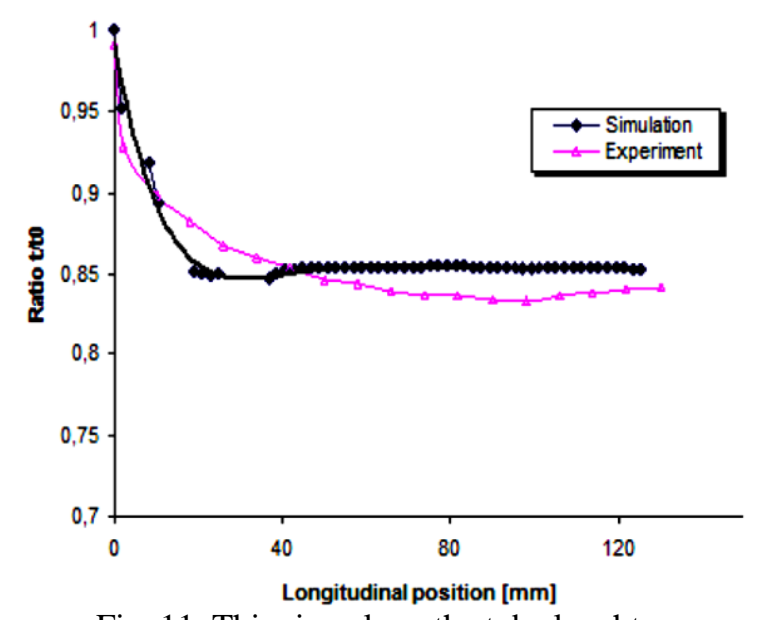

Fig. 11. Thinning along the tube lenght

\section{Conclusion}

The paper presents the results of a combined experimental/numerical effort that aims to assess the performance of different plastic stress flow in predicting burst of welded steel tubes loaded under internal pressure. The prediction of the stressstrain characteristic with anisotropic effect of tubular material has been proposed. Once the expanding diameter, the internal pressure and the wall thickness were obtained from the results of the bulge tests, the effective stress and effective strain could be calculated. The bulge tests carried out until bursting showed that all the fissures are 
initiated on the central area of the expanding zone not far from the weld zone. This failure can be attributed to the geometrical singularities of the WJ and the inhomogeneous behaviour of the BM and the HAZ.

Using the least square method, a flow stress curve that best fits the stress-strain of the BM material with geometrical singularities could be determined with and without consideration of anisotropy. The local behaviour (stress/strain) of the welded joints and the HAZ is identified numerically using Abaqus solver from global results (force/depth) of nanoindentation tests. The identified hardening coefficients are introduced by Swift model. From the simulations carried out, it is clear the influence of the plastic flow behaviour of the WT in the final results (thickness distribution, stress instability, tube circularity and critical thinning and rupture).

It is also clear that to predict with more accuracy the results, the model used for simulation has to be as realistic as possible. Therefore, future work in this area will include the experimental identification approach of the hardening model coupled with damage. Indeed, we think that measurements of displacements and strains without contact can improve results quality. The suggested model coupled with ductile damage can contribute to the deduction of forming limit diagrams

\section{References}

1. F. Dohmann, C. Hertl, Tube hydroformingresearch and practical application, Journal of Materials Processing Technology, 71:174186, (1997).

2. Y. Jae-Bong, J. Byung, O. Soo, The tube bending technology of a hydroforming process for an automotive part, Journal of Materials Processing Technology, 111: 175-181, (2001).

3. Y.S. Shin, H.Y. Kim, B.H. Jeon, S.I. Oh, Prototype tryout and design for automotive parts using welded blank hydro forming, J Mater Process Techno, 130-131:121-127, (2002).

4. R.M. Natal Jorge, A.P. Roque, R.A.F. Valente, M.P.L. Parente and A. Fernandes, $A$ Study of hydro formed tailor-welded tubular parts with dissimilar thickness, J Mater Process Techno, 184:363-371, (2007).

5. G. Liu, S. Yuan and G. Chu, FEA on deformation behavior of tailor-welded tube in hydroforming, J Mater Process Techno, 187188:287-291, (2007).

6. J.P. Abrantes, A. Szabo-Ponce, G.F. Batalha, Experimental and numerical simulation of tube hydroforming (THF), J Mater Process Techno, 164-165: 1140-1147, (2005).

7. L. Lang, S. Yuan, X. Wang, Z.R. Wang, Z. $\mathrm{Fu}$, J. Danckert and K.B. Nielsen, A study on numerical simulation of hydroforming of aluminum alloy tube, J Mater Process Techno, 146:377-388, (2004).

8. M. Ayadi, H. Cherouat, N. Mezghani and M.A. Rezgui, Experimental and numerical studies of welded tube formability, $6^{\text {th }}$ International Forum on Advanced Material Science and Technology, Hong Kong, June 12-14, (2008).

9. G. Liu, S. Yuan and G. Chu, FEA on deformation behavior of tailor-welded tube in hydroforming, J Mater Process Techno, 187188:287-291, (2007).

10. N. Mezghani, H. Salhi, M. Ayadi, A. Cherouat, Experimental and numerical simulation of hydroforming process, International Review of Mechanical Engineering, to appear (2009).

11. M. Imaninejad, G. Subhash and A. Loukus, Influence of end conditions during tube hydro forming of aluminum extrusions, I J Mech Scien, 46:1195-1212, (2004).

12. P. Ray and B.J. Mac Donald, Determination of the optimal load path for tube hydro forming processes using a fuzzy load algorithm and finite element analysis, Finite element in Analysis and Design, 41:173-192, (2004).

13. G. Neffussi, A. Combescure, Coupled buckling and plastic instability for tube hydroforming, I J Mech Scien, 44:899-914, (2002).

14. K.I. Johnson, B.N. Nguyen, R.W. Davies, G.J. Grant and M.A. Khaleel, A numerical process control method for circular tube hydroforming prediction, I J Plasticity, 20:1111-1137, (2004).

15. S. Yuan, W. Yuan and X. Wang, Effect of wrinkling behavior on formability and thickness distribution in tube hydroforming, $\mathrm{J}$ Mater Process Techno, 177:668-671, (2006). 
16. M. Koç, Investigation of the effect of loading path and variation in materiel proprieties on robustness for tube hydro forming, $\mathrm{J}$ Mater Process Techno, 133:276-281, (2003).

17. K-J Fann, P-Y Hsiao, Optimization of loading conditions for tube hydroforming, $\mathrm{J}$ Mater Process Techno, 140:520-524, (2003).

18. K. Manabe, M. Suetake, H. Koyama and M. Yang, Hydroforming process optimization of aluminum alloy tube using intelligent control technique, I J Machine Tools \& Manuf, 46:1207-1211, (2006).

19. T. Hama, T. Ohkubo, K. Kurisu, H. Fujimoto and H. Takuda, Formability of tube hydroforming under various loading paths, J Mater Process Techno, 177:676-679, (2006).

20. R.M. Natal Jorge, A.P. Roque, R.A.F. Valente, M.P.L. Parente and A. Fernandes, A Study of hydro formed tailor-welded tubular parts with dissimilar thickness, $\mathrm{J}$ Mater Process Techno, 184:363-371, (2007).

21. A. Cherouat, K. Saanouni, Y. Hammi, Numerical improvement of thin tubes hydroforming with respect to ductile damage, I J Mech Scien., 44:2427-2446, (2002).
22. M. Imaninejad, G. Subhash, A. Loukus, Experimental and numerical investigation of free-bulge formation during hydroforming of aluminium extrusions, J Mater Process Techno, 147:247-254, (2004).

23. P. Bortot, E. Ceretti, C. Giardini, The determination of flow stress of tubular material for hydroforming, $\mathrm{J}$ Mater Process Techno, 203: 381-388, (2008).

24. C. Levaillant, J.L. Chenot, Physical modelling and numerical prediction of defects in sheet metal forming, J Mater Process Techno, 32:383-397, (1992).

25. P. Hora, L. Tong, J.A. Reissner, Prediction method for ductile sheet metal failure in $\mathrm{Fe}$ simulation, Wagonner et al. Eds., Proceedings of Numisheet'96 :252-256, (1996).

26. X. Liang, Stress based fracture envelope for damage plastic solids, Engineering Fracture Mechanics, 76:419-438, (2009). 